\title{
Drs2 flips the phospholipid switch
}

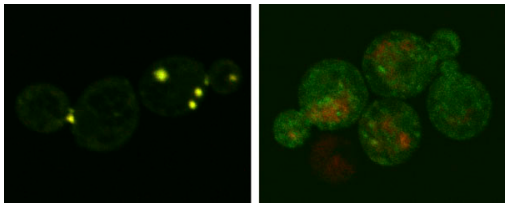

Gcs 1 (green) concentrates in the transGolgi network and early endosomes (red) in control cells (left), but, in cells lacking Drs2 (right), the protein is widely dispersed.

he protein Drs2 flips phospholipids between membrane layers. Xu et al. identify which phospholipid the protein relocates to allow transport between the transGolgi network and the early endosome.

Drs2 commutes from the trans-Golgi network (TGN) to the early endosome (EE) and then back again. Without Drs2, cells can't ship proteins along this route. The molecule is a flippase and moves phospholipids from the inner (lumenal) to the outer (cytosolic) leaflets of membranes. Insertion of a phospholipid into the cytosolic leaflet bends the membrane, which may help spur budding of new transport vesicles. Drs2 can flip the phospholipids phosphatidylserine and phosphatidylethanolamine,

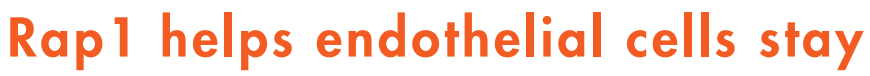

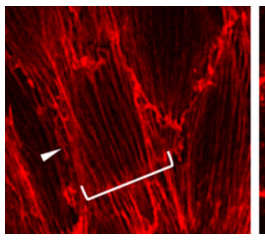

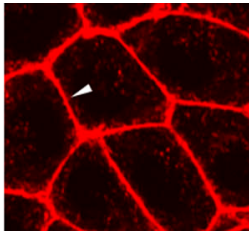

Endothelial cells can show loose connections (left), but the cells can also form tighter junctions (right) delineated by bundles of actin (red).

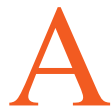

ndo et al. reveal a molecular pathway that tightens the junctions between endothelial cells.

Endothelial junctions must be flexible. To allow white blood cells to exit from a capillary, for example, endothelial cells need to loosen the connections between them. Two types of junctions in endothelial layers provide this adaptability. Zonula adherens are tighter, stable connections that are strengthened by clusters of actin fibers, called circumferential actin bundles (CABs), that run alongside the junctions. Smaller punctate junctions are looser and are bolstered by radial stress fibers

\section{Silicon nanocrystals shine}
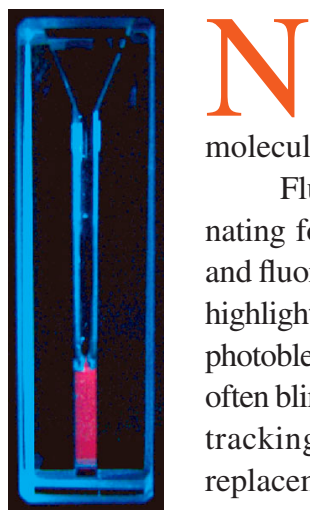

ishimura et al. describe silicon nanocrystals that overcome some of the failings of fluorescent proteins and similar molecular labels.

Fluorescence microscopy has been illuminating for cell biologists, but probes such as GFP and fluorescent organic compounds that are used to highlight molecules have some drawbacks. The tags photobleach, or fade when exposed to light, and they often blink. These limitations make single-molecule tracking difficult. Quantum dots are possible replacements, but they are bulky. Silicon nano-

A tube of silicon nanocrystals glows red after being stimulated with ultraviolet light. crystals seem like an ideal alternative because they can be smaller than GFP, rarely photobleach, and can emit several different wavelengths of light. However, but researchers weren't sure which proteins help Drs2 shift phospholipids and which type of phospholipid it moves to enable transport between the TGN and EEs.

$\mathrm{Xu}$ et al. revealed that Drs2 partners with the protein Gcs1, which is necessary for transport from the EE to the TGN, and lures Gcs 1 to the EE and TGN. Gcs 1 carries a motif, called ALPS, that recognizes bent membranes. However, the protein required a slightly longer version of the motif, which the team dubbed +ALPS, to attach to the EE and TGN. Transferring certain phospholipids into the outer leaflet not only bends the membrane, it also increases the negative charge on the surface. The lengthened ALPS sequence enables Gcs1 to recognize the bowed, negatively charged section of membrane created by Drs2.

By testing a mutant version of Drs2 that can't grab phosphatidylserine but can attach to phosphatidylethanolamine, the team discovered that the protein relocates phosphatidylserine to promote transportation between the EE and TGN.

Xu, P., et al. 2013. J. Cell Biol. http://dx.doi.org/10.1083/jcb.201305094.

\section{close}

that run perpendicular to the junctions. The GTPase Rap1 spurs the formation of CABs, but researchers weren't sure how.

Ando et al. discovered that Rap1 spurs CAB formation by stimulating non-muscle myosin II. One way to activate this myosin is through the Rho-ROCK pathway, but the team found that this pathway triggers formation of radial stress fibers, not CABs. Instead, Rap1 activates the kinase MRCK to switch on myosin II at cell junctions.

The researchers then teased out more details of this pathway. Rap1 turns on $\mathrm{Cdc} 42$ and directs it to the cell-cell contacts. In turn, Cdc42 draws MRCK to the junctions. Rap1 also inhibits the Rho-ROCK pathway, preventing it from turning on myosin II in the cytoplasm and inducing radial stress fibers. Activating Rap1 solidified connections between cultured endothelial cells, suggesting that the pathway spurs formation of zonula adherens.

Ando, K., et al. 2013. J. Cell Biol. http://dx.doi.org/10.1083/jcb.201301115.

researchers have had difficulty making uniform particles and attaching them to individual molecules.

Nishimura et al. used a novel technique that involves chemically etching silicon wafers. The researchers crafted uniform silicon nanocrystals that emitted red light and were $4.1 \mathrm{~nm}$ in diametersmaller than a single GFP molecule. During the five hours the team observed them, the crystals didn't photobleach or blink.

After coupling transferrin molecules to single nanocrystals, the researchers tracked individual transferrin receptors in the plasma membrane of kidney cells. Because fluorescent tags blink, researchers are often forced to interpolate a molecule's trajectory during the outage. With this new system, the team was able to follow nanocrystal-tagged molecules continuously for two minutes, compared with less than a second for GFP-labeled molecules. The researchers are now working to develop crystals that emit different wavelengths of light.

Nishimura, H., et al. 2013. J. Cell Biol. http://dx.doi.org/10.1083/icb.201301053. 